\title{
Phosphorylation of Snapin by PKA modulates its interaction with the SNARE complex
}

\author{
Milan G. Chheda*, Uri Ashery†, Pratima Thakur†, Jens Rettig†‡ and Zu-Hang Sheng ${ }^{\text {}}$ \\ *Synaptic Function Unit, National Institute of Neurological Disorders and Stroke, National Institutes of Health, 36 Convent Drive, Bethesda, Maryland 20892-4154, USA \\ †Department of Membrane Biophysics, Max Planck Institute for Biophysical Chemistry, Am Fassberg, 37077 Göttingen, Germany \\ ‡Current address: Physiologisches Institut, Universität des Saarlandes, 66421 Homburg, Germany \\ Se-mail: shengz@ninds.nih.gov
}

\begin{abstract}
cAMP-dependent protein kinase $\mathrm{A}$ (PKA) can modulate synaptic transmission by acting directly on unknown targets in the neurotransmitter secretory machinery. Here we identify Snapin, a protein of relative molecular mass 15,000 that is implicated in neurotransmission by binding to SNAP-25, as a possible target. Deletion mutation and sitedirected mutagenetic experiments pinpoint the phosphorylation site to serine 50. PKA-phosphorylation of Snapin significantly increases its binding to synaptosomal-associated protein-25 (SNAP-25). Mutation of Snapin serine 50 to aspartic acid (S50D) mimics this effect of PKA phosphorylation and enhances the association of synaptotagmin with the soluble $\mathrm{N}$-ethylmaleimide-sensitive factor attachment protein receptor (SNARE) complex. Furthermore, treatment of rat hippocampal slices with nonhydrolysable cAMP analogue induces in vivo phosphorylation of Snapin and enhances the interaction of both Snapin and synaptotagmin with the SNARE complex. In adrenal chromaffin cells, overexpression of the Snapin S50D mutant leads to an increase in the number of release-competent vesicles. Our results indicate that Snapin may be a PKA target for modulating transmitter release through the cAMP-dependent signal-transduction pathway.
\end{abstract}

D ocking and fusion of synaptic vesicles at release sites requires the association of proteins on both vesicle and plasma membranes ${ }^{1-4}$. The synaptic-vesicle-associated protein synaptobrevin/VAMP interacts with the plasma membrane associated proteins SNAP-25 and syntaxin, constituting the SNARE complex, a biochemical intermediate that is essential for vesicular transport and fusion processes ${ }^{5-10}$. Formation of the SNARE complex is the minimum molecular requirement for membrane fusion in vitro ${ }^{11,12}$. Synaptotagmin, which is present in synaptic-vesicle membranes, binds to $\mathrm{Ca}^{2+}$ at nerve terminals and presumably triggers neurotransmitter release by interacting with the SNARE complex ${ }^{13-15}$.

Synaptic transmission is modulated by pre- and postsynaptic mechanisms, some of which are involved in synaptic plasticity and memory ${ }^{16-19}$. Second-messenger regulation of protein interactions within the exocytotic apparatus is potentially important in synaptic plasticity3. Second-messenger-activated protein kinases expressed in presynaptic terminals include PKA, calcium/phospholipid-dependent protein kinase (PKC), calcium- and calmodulin-dependent protein kinase II (CaM KII), and cGMP-dependent protein kinase (PKG). At many synapses, presynaptic activation of PKA can increase the amount of neurotransmitter released per action potential ${ }^{20-23}$. The cAMP/PKA cascade augments vesicular release at synapses between cerebellar granule cells in primary culture ${ }^{24}$. Long-term potentiation (LTP) in the mossy-fibre pathway in the hippocampal CA3 region seems to be a presynaptic mechanism that is mediated by cAMP-PKA signalling ${ }^{25,26}$. In hippocampal neurons, activation of PKA increases neurotransmitter release by acting directly on the exocytotic apparatus, rather than by enhancing docking of morphologically recognizable vesicles or by increasing the number of functional terminals ${ }^{27}$. In addition, PKA modulation either increases the cooperativity of $\mathrm{Ca}^{2+}$ for release or enhances the interaction between the $\mathrm{Ca}^{2+}$-sensing module and the exocytotic apparatus ${ }^{28}$. Together, this evidence indicates that one or more regulatory proteins in the synaptic vesicle fusion complex can be phosphorylated by PKA, and that this phosphorylation may regulate the exocytotic process. Thus, identifying the PKA target(s) that regulate(s) assembly/disassembly of the fusion machinery is critical to elucidate the molecular mechanisms that underlie synaptic facilitation.

Recent electrophysiological studies of the effect of Botulinum A (BontA) toxin on hippocampal neurons have led to the dual predictions that PKA modulates an early, calcium-sensing step in the secretory process that facilitates synaptic transmission, and that either SNAP-25 or a protein (or proteins) with which it interacts is required for this PKA-dependent modulation ${ }^{28}$. Here we show that Snapin is one such PKA target. Snapin is a newly discovered protein that is present in the membranes of synaptic vesicles and is implicated in neurotransmission by directly binding to SNAP-25 (ref. 29). Here we show that both recombinant and native Snapin derived from rat-brain synaptosomes can be phosphorylated by PKA. PKA phosphorylation of Snapin significantly increases its binding to SNAP-25, and consequently enhances the association of synaptotagmin with the SNARE complex, a key step during $\mathrm{Ca}^{2+}$ dependent exocytosis. In adrenal chromaffin cells, overexpression of the Snapin S50D mutant, which mimics a state of complete phosphorylation of the native protein, leads to an increase in both the number of release-competent vesicles and the kinetics of the priming reaction. Our results indicate that Snapin is a physiologically significant PKA target in synapses.

\section{Results}

In vitro phosphorylation of recombinant and native Snapin. As it has been predicted that either SNAP-25 or a protein with which it interacts is a target of $\mathrm{PKA}^{28}$, and as Snapin is known to bind to SNAP-25 and increase the association of synaptotagmin with the SNARE complex $^{29}$, we wondered whether the Snapin-SNAP-25 interaction could be regulated by PKA. First, we examined the ability of recombinant Snapin to serve as a substrate for PKA. We incubated 100 pmol of purified full-length Snapin, tagged with histidine 

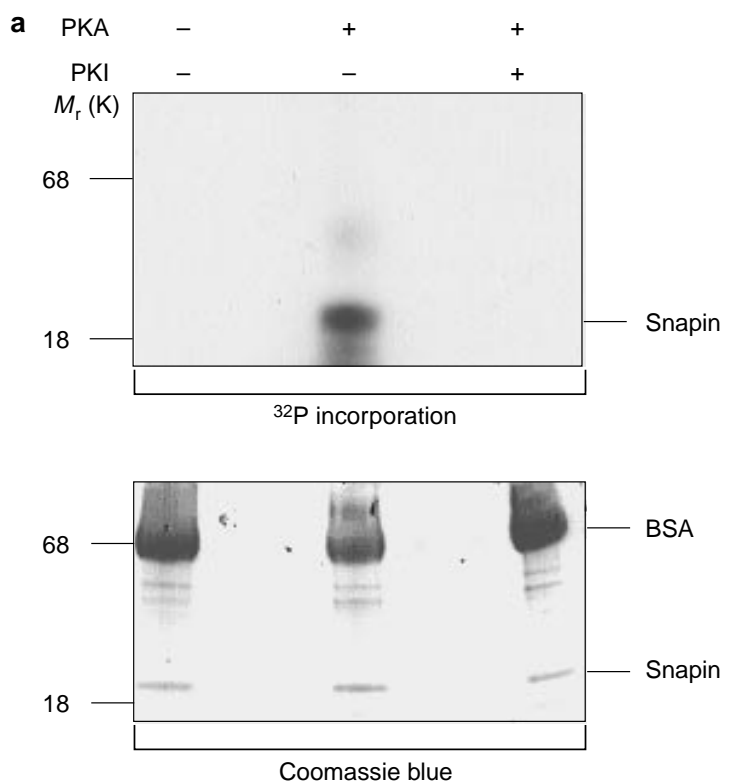

b

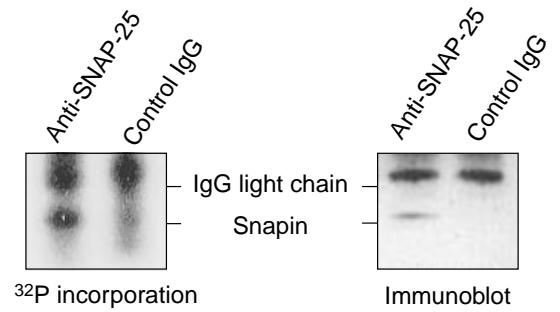

Figure 1 PKA-dependent phosphorylation of Snapin in vitro. a,

Phosphorylation of recombinant Snapin by PKA. Full-length Snapin (100 pmol) tagged with histidine was incubated with [ $\gamma^{32}$ P]ATP in a $35-\mu$ reaction in the presence $(+)$ or absence $(-)$ of the catalytic subunit of PKA or PKA-inhibitor peptide (PKI). Phosphorylation products were separated by $10-20 \%$ Tricine SDS-PAGE; gels were then stained with Coomassie blue, dried, and exposed to X-ray film. Relative molecular masses $\left(M_{\mathrm{r}}\right)$ are indicated on the left. $\mathbf{b}$, Phosphorylation by PKA of native Snapin from solubilized synaptosomes. Native Snapin was recovered from solubilized rat-brain synaptosomes by immunoprecipitation with an anti-SNAP-25 antibody. Immunoprecipitates were subjected to phosphorylation in the presence of the catalytic subunit of PKA. Phosphorylation products were then separated by $16 \%$ SDS-PAGE and processed by autoradiography (left panel). To confirm the identity of the ${ }^{32 P}$-containing band as Snapin, co-precipitates were analysed by immunoblotting with an anti-Snapin antibody (right panel).

(His), with $\left[\gamma_{-}{ }^{32} \mathrm{P}\right]$ ATP and the catalytic subunit of PKA. We terminated reactions by adding SDS sample buffer and heating, and separated the products on 10-20\% Tricine SDS-polyacrylamide gel, which we stained with Coomassie blue to verify equal amounts of protein loaded on each lane. We then dried the gel and exposed it to $\mathrm{X}$-rays to detect ${ }^{32} \mathrm{P}$ incorporation. As shown in Fig. 1a, Snapin functions as a substrate for PKA, and its phosphorylation is blocked by the pseudosubstrate peptide inhibitor PKI. As controls, we also incubated Snapin with PKC or CaM KII, together with their cognate activators. Under our reaction conditions, weak PKC-dependent incorporation of ${ }^{32} \mathrm{P}$ into Snapin was detected after prolonged autoradiography, whereas CaM KII-dependent ${ }^{32} \mathrm{P}$ incorporation was not detectable (data not shown).

Our previous study ${ }^{29}$ showed that Snapin is associated with the SNARE complex in synaptosome preparations. To determine whether native Snapin associated with the SNARE complex is a substrate for PKA, we co-immunoprecipiatated Snapin-SNARE
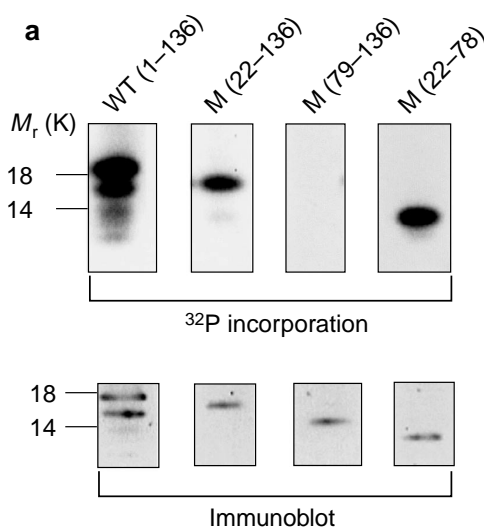

b
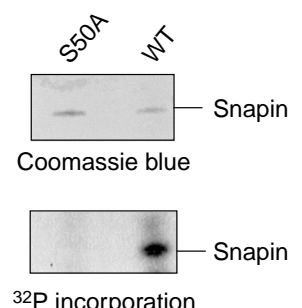

Figure 2 Serine $\mathbf{5 0}$ is the site of PKA phosphorylation in Snapin. a,

Phosphorylation of Snapin deletion mutants by PKA. Roughly 50 pmol of wild-type (WT) and the indicated deletion-mutant (M) His-tagged recombinant Snapin proteins were phosphorylated by PKA (see Methods). Reaction products were separated by 10-20\% Tricine SDS-PAGE; gels were then dried and exposed to X-ray film for autoradiography (upper panel). Mutant Snapin proteins were further confirmed by immunoblotting with anti-His antibody (lower panel). Relative molecular masses $\left(M_{r}\right)$ are shown on the left. $\mathbf{b}$, Phosphorylation of wild-type and S50A Snapin by PKA. Proteins (50 pmol each) were incubated with PKA-phosphorylation reagents and products were separated by 10-20\% SDS-PAGE; gels were then stained with Coomassie blue (upper panel), dried, and exposed to X-ray film for autoradiography (lower panel).

complexes with an anti-SNAP-25 antibody from detergent extracts of rat-brain synaptosomes and analysed the precipitates for in vitro PKA phosphorylation. As shown in Fig. 1b, native Snapin can be phosphorylated. We further confirmed the specificity of Snapin for the ${ }^{32} \mathrm{P}$-containing band by immunoblotting co-precipitates with an anti-Snapin antibody. These data indicate that the phosphorylation site on native Snapin is accessible to PKA while it is associated with SNAP-25, and that Snapin is a potential PKA target in the vesicle exocytotic apparatus of synapses.

Mapping the PKA phosphorylation site. To map potential PKA phosphorylation sites, we first examined the capacity of Snapin deletion mutants to serve as PKA substrates. We incubated $\sim 50 \mathrm{pmol}$ of full-length wild-type or deletion-mutant Snapin proteins, tagged with His, with the catalytic subunit of PKA. We separated the products using 10-20\% Tricine SDS-polyacrylamide gel, which we then either dried and then exposed to X-ray film for autoradiography, or immunoblotted with an anti-His antibody. As shown in Fig. 2a, the amino-terminal fragment comprising residues $22-78$ was efficiently phosphorylated in vitro by PKA. In contrast, the carboxy-terminal fragment of residues 79-136 was not a PKA substrate. The sequence of residues 22-78 contains three serine/threonine residues. To identify the amino-acid residue(s) phosphorylated by PKA, we used sitedirected mutagenesis to generate three Snapin mutants in which the serine at position 42 or 50 or the threonine at position 63 was substituted with alanine. Whereas both S42A and T63A mutants were efficient substrates of PKA (data not shown), the S50A mutation 
a

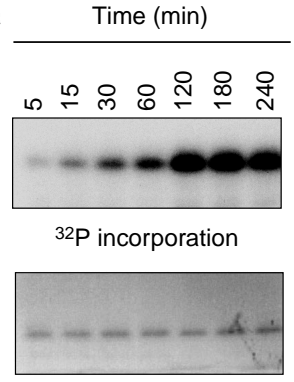

Coomassie blue

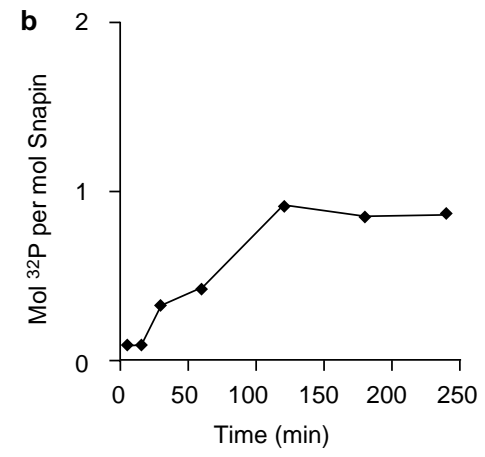

Figure 3 Stoichiometry of PKA phosphorylation of Snapin. Snapin was phosphorylated by PKA for the indicated time periods. Reactions were terminated by addition of SDS sample buffer and boiling; products were separated by SDS-PAGE and the gel was stained with Coomassie blue and exposed to X-ray film for $1 \mathrm{~h} \mathrm{(a)}$. Gel slices containing labelled Snapin were excised and scintillation-counted, and the molar amount of ${ }^{32} \mathrm{P}$ incorporated per mol Snapin were calculated and plotted as a function of time (b).

effectively eliminated ${ }^{32} \mathrm{P}$ incorporation (Fig.2b), indicating that serine 50 is the primary site of PKA phosphorylation. The sequence of the surrounding residues (VRES-50) matches one of the consensus sequences for PKA (RRXS/T, RXS/T, and $\mathrm{RX}_{2} \mathrm{~S} / \mathrm{T}$; ref. 30).

To confirm further that PKA phosphorylates only one residue in Snapin, we carried out a stoichiometric analysis of phosphorylation for purified recombinant Snapin protein. The reactions included equal amounts $(15 \mathrm{pmol})$ of Snapin and excess PKA and $[\gamma$ ${ }^{32} \mathrm{P}$ ]ATP, and were terminated at various time points by addition of SDS sample buffer and boiling. We separated the products using SDS-polyacrylamide gel, which we stained with Coomassie blue to verify equal amounts of protein loaded on each lane. We then dried the gel and sbjected it to autoradiography for $1 \mathrm{~h}$ (Fig. 3a). To quantify ${ }^{32} \mathrm{P}$ incorporation, we excised gel slices corresponding to phosphorylated Snapin bands and subjeted them to scintillation counting. The results are shown in Fig. 3b; stoichiometry values are expressed as the ratio of $\mathrm{mol}^{32} \mathrm{P}$ incorporated per mol Snapin and are plotted against reaction time. The maximal stoichiometries of $\sim 1$ are consistent with the identification of a single site of PKA phosphorylation in the mutagenesis experiments.

Functional consequences of Snapin phosphorylation by PKA. It has been reported that SNAP-25 is PKA-phosphorylated at threonine 138 , and that the phosphorylated state of SNAP-25 does not regulate the assembly or stability of the ternary SNARE complex ${ }^{31,32}$. However, one contradictory report argues that SNAP-25 is not a substrate for PKA in vitro ${ }^{33}$. To investigate whether phosphorylated SNAP-25 has any effect on binding to Snapin, we first repeated the PKA-phosphorylation studies using recombinant SNAP-25. We incubated $\sim 50$ pmol of purified SNAP- 25 or Snapin together with the catalytic subunit of PKA. SNAP-25 was phosphorylated by PKA to a similar extent as was Snapin (data not shown).

We next investigated the effects of PKA phosphorylation of Snapin or SNAP-25 on their interactions. We incubated glutathioneS-transferase (GST) beads with immobilized PKA-phosphorylated or unphosphorylated SNAP-25, together with 25 pmol of PKAphosphorylated or unphosphorylated Snapin. After incubation for 5 $\mathrm{h}$ at $4{ }^{\circ} \mathrm{C}$, we washed the beads, separated the resulting protein complexes by SDS-polyacrylamide-gel electrophoresis (PAGE), and sequentially probed them with anti-Snapin and anti-GST antibodies. Whereas PKA phosphorylation of SNAP-25 did not affect its binding, phosphorylated Snapin exhibited significantly increased binding to SNAP-25 relative to the unphosphorylated control (Fig. 4a).

Our mutagenetic studies showed serine 50 to be the primary site for PKA phosphorylation of Snapin. To determine whether a
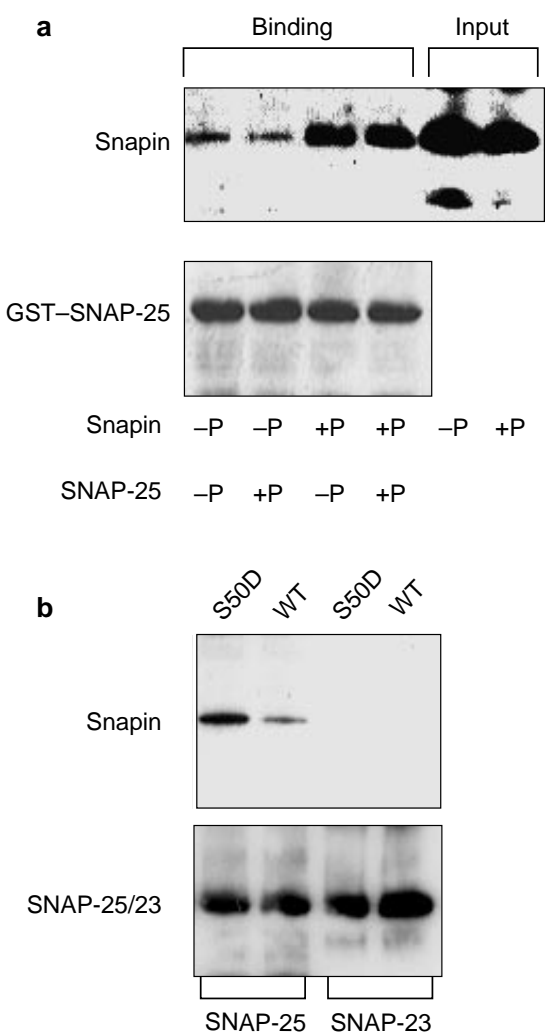

Figure 4 Functional consequences of phosphorylation of Snapin by PKA. a, Phosphorylation of Snapin by PKA increases its binding to SNAP-25. GST beads with immobilized SNAP-25 were incubated with phosphorylation buffer in the presence $(+P)$ or absence (-P) of the catalytic subunit of PKA. After extensive washing, $\sim 25$ pmol of PKA-phosphorylated (+P) or unphosphorylated (-P) Snapin were added to GST beads. After incubation for $5 \mathrm{~h}$ at $4{ }^{\circ} \mathrm{C}$, beads were washed, and the GST-SNAP-25-Snapin complex was separated by SDS-PAGE, blotted on nitrocellulose, and probed with an anti-Snapin antibody (upper panel). Membranes were then stripped and reprobed with an anti-GST antibody (lower panel). Bound antibodies were detected by enhanced chemiluminescence. Results are representative of three independent experiments. b, Replacing serine 50 with aspartic acid mimics the effect of the PKA phosphorylation of Snapin on its binding to SNAP-25. Roughly $37 \mathrm{pmol}$ of full-length wild-type (WT) or S50D Snapin were incubated with an equal amount of GST-SNAP-25 or SNAP-23 immobilized on sepharose beads for $5 \mathrm{~h}$ at 4 ${ }^{\circ} \mathrm{C}$. The resulting complexes were separated by SDS-PAGE, transferred to nitrocellulose, and probed with anti-Snapin antibody (upper panel). Membranes were then stripped and reprobed with anti-GST antibody (lower panel). Binding intensities were quantified using NIH Image scanning. The relative levels of binding of wild-type and S50D Snapin to GST-SNAP-25 were calculated using a linear standard curve of Snapin aliquots. Semi-quantitative analysis revealed that the S50D mutant elicited a 3.5-fold increase in binding to SNAP-25 relative to that of wild-type Snapin.

introduction of a negatively charged residue at this site is sufficient to account for the increased binding of phosphorylated Snapin to SNAP-25, we attempted to mimic complete phosphorylation of serine 50 by mutating it to negatively charged aspartic acid (S50D) and analysing the resultant binding properties of the protein. We incubated $\sim 37$ pmol of full-length wild-type or S50D Snapin with equal amounts of GST-SNAP-25 immobilized on sepharose beads for $5 \mathrm{~h}$ at $4{ }^{\circ} \mathrm{C}$. As a control, we included GST-SNAP-23, which does not interact with Snapin ${ }^{29}$, in these in vitro binding assays. We separated protein complexes by SDS-PAGE, and sequentially probed the cognate membrane with anti-Snapin and anti-GST antibodies. The S50D mutation mimicked the effect of PKA phosphorylation of Snapin on its binding to SNAP-25 (Fig. 4b). Semi-quantitative 


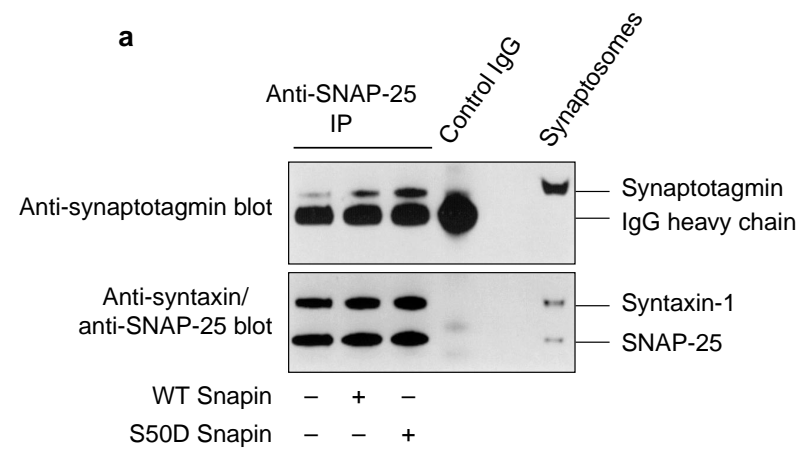

b

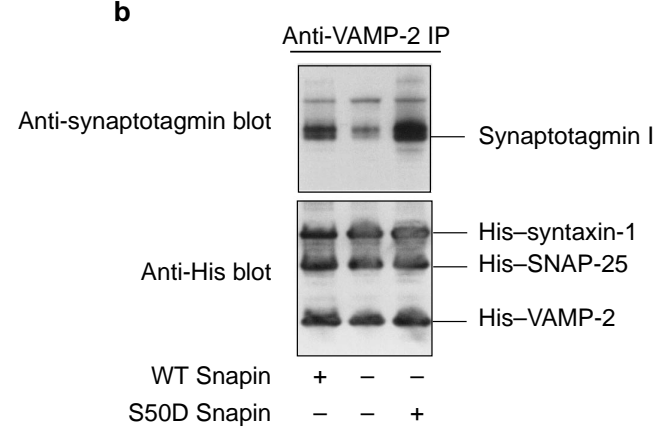

c

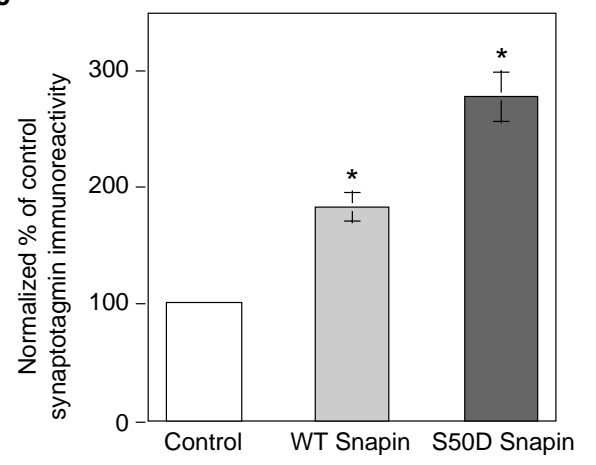

Figure 5 Effect of the S50D mutant on the association of synaptotagmin with the SNARE complex. a, Immunoprecipitation (IP) of native SNARE proteins with synaptotagmin I. An anti-SNAP-25 antibody immunoprecipitated synaptotagmin with SNARE proteins from solubilized rat-brain synaptosomes in the presence or absence of $66 \mathrm{pmol}$ of wild-type or S50D Snapin. Co-immunoprecipitates were analysed by sequential immunoblotting of the same membrane after stripping of previous antibodies. The right-most lane was loaded with solubilized synaptosomes as a detection control for antibodies. Note that the association of synaptotagmin with the SNARE complex is significantly increased by co-incubation with the S50D mutant $(181 \pm 23 \%, n=3, P<0.05)$ relative to wild-type Snapin, whereas the amount of SNARE core proteins (syntaxin-1, SNAP-25) co-precipitated remains unchanged. $\mathbf{b}$, Immunoprecipitation of recombinant SNARE proteins with synaptotagmin I. An anti-VAMP-2 antibody was co-incubated with purified recombinant synaptotagmin, His-SNAP-25, His-syntaxin-1A and His-VAMP-2 (100 pmol each) in the presence or absence of $110 \mathrm{pmol}$ of wild type or S50D Snapin. Co-immunoprecipitates were analysed by sequential immunoblotting with anti-synaptotagmin and anti-His antibodies. c, Normalized amounts of recombinant synaptotagmin I that coimmunoprecipitated as shown in $\mathbf{b}$. Values are means \pm s.e.m. from four experiments; asterisk denotes $P<0.05$.

analysis revealed that this mutation elicited a 3.5-fold increase in binding to SNAP- $25(347 \pm 32 \%, n=6, P<0.01)$ relative to wildtype Snapin.
We previously reported that Snapin functions in the neurotransmitter-release process through modulation of the sequential interactions between SNARE proteins and synaptotagmin ${ }^{29}$. To understand further the role of Snapin in neurotransmitter release, we investigated whether the S50D mutant, which mimics a completely phosphorylated state, can influence the association of the SNARE complex with synaptotagmin. We incubated solubilized proteins from rat-brain synaptosomes with anti-SNAP-25 antibody in the presence or absence of wild-type or S50D Snapin (66 pmol each). After capture using protein A-sepharose, we subjected the resulting complexes to SDS-PAGE and detected proteins by sequentially immunoblotting the same membrane for synaptotagmin, syntaxin-1 and SNAP-25. Whereas wild-type Snapin increased the amount of association of synaptotagmin with the SNARE complex, S50D Snapin potentiated this association to an even greater extent (181 $\pm 23 \%, n=3, P<0.05$; Fig.5a). No immunoprecipitation was observed in the presence of a mouse immunoglobulin G control.

To exclude the possibility that the increased association of synaptotagmin with SNAREs after PKA phosphorylation was due to the presence of other proteins in synaptosomal preparations, we extended our co-immunoprecipitation study by using purified recombinant proteins. We incubated an anti-VAMP-2 antibody with purified recombinant synaptotagmin, His-SNAP-25, His-syntaxin$1 \mathrm{~A}$ and His-VAMP-2 (100 pmol each) in the presence or absence of 110 pmol of wild-type or S50D Snapin. We analysed co-immunoprecipitates by sequential immunoblotting with anti-synaptotagmin and anti-His antibodies. Once again, whereas wild-type Snapin increased the amount of association of synaptotagmin with recombinant SNARE complex $(183 \pm 12 \%, n=4)$, S50D Snapin potentiated this association to a greater extent $(277 \pm 20 \%, n=4, P<0.05)$ relative to control in the absence of Snapin (Fig. 5b, c). Neither wildtype nor mutant Snapin affected the binding affinity of SNARE proteins, as roughly equal amounts of syntaxin-1, VAMP-2 and SNAP25 were detected (Fig. 5b). Thus, our co-immunoprecipitation studies using native and recombinant proteins indicate that the interaction of synaptotagmin with the SNARE complex is significantly increased after addition of S50D Snapin.

To determine the cellular significance of phosphorylation of Snapin by PKA in neurons, we first investigated whether Snapin can be phosphorylated through a cAMP-dependent mechanism in vivo. Although our biochemical experiments have shown that purified PKA incorporates ${ }^{32} \mathrm{P}$ into both recombinant and native Snapin (Fig. 1), in vitro phosphorylation conditions may not reflect the native cell environment. In addition, solubilization and immunoprecipitation may expose a site that is normally not available for phosphorylation in vivo. Therefore, to investigate in vivo phosphorylation, we carried out back-phosphorylation assays. In this procedure, endogenous phosphate is incorporated in vivo into Snapin after stimulation with PKA activator in rat hippocampal slices. Cell lysates from stimulated and unstimulated hippocampal slices are in vitro-phosphorylated (back-phosphorylation) with purified PKA to incorporate ${ }^{32} \mathrm{P}$ into Snapin that was left unphosphorylated after stimulation. In this protocol, a reduction in back-phosphorylation reflects an increase in in vivo phosphorylation of Snapin by endogenous PKA. To activate PKA in hippocampal neurons, we used a relatively new nonhydrolysable cAMP analogue, Sp-5,6DCl-cBIMPS (BIMPS), which is better than its parent compound, Sp-cAMPS, in several respects, including higher metabolic stability, greater lipophilicity and excellent membrane permeability ${ }^{34}$. We incubated hippocampal slices with $50 \mu \mathrm{M}$ BIMPS for $30 \mathrm{~min}$ at 37 ${ }^{\circ} \mathrm{C}$, and solubilized them with $1.2 \%$ Triton X-100. As expected for a direct phosphorylation mechanism on Snapin, the amount of ${ }^{32} \mathrm{P}$ incorporated during back-phosphorylation was significantly reduced when hippocampal slices were stimulated in vivo with BIMPS (Fig. 6a, upper panel). Immunoblots (Fig. 6a, lower panel) using an antibody against Snapin confirmed the identity of the phosphorylated band (relative molecular mass 15,000) as Snapin and served to normalize back-phosphorylation to the amount of 

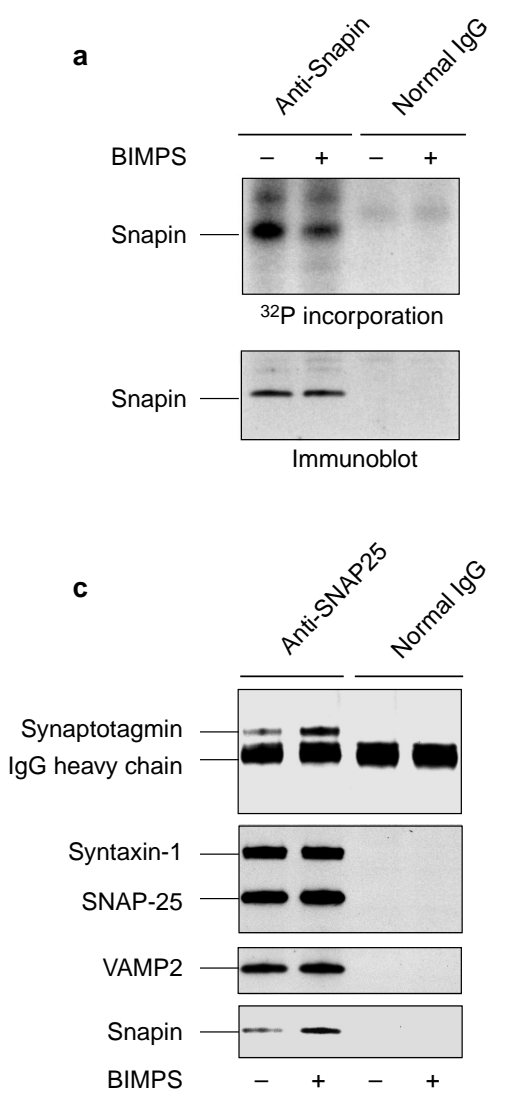

Figure 6 cAMP-dependent phosphorylation of Snapin potentiates interactions of Snapin and synaptotagmin with the SNARE complex. a, In vivo CAMPdependent phosphorylation of Snapin. Rat hippocampal slices were incubated with $50 \mu \mathrm{M}$ BIMPS at $37^{\circ} \mathrm{C}$ for $30 \mathrm{~min}$ and then solubilized with Triton X-100. Total protein $(150 \mu \mathrm{g})$ was incubated with an anti-Snapin antibody or normal immunoglobulin $\mathrm{G}$ (IgG) control. Immunoprecipitates were then back-phosphorylated in vitro with purified PKA. Proteins were resolved by SDS-PAGE and were then autoradiographed (upper panel), or immunoblotted (lower panel). b, Normalized amount of back-phosphorylation in unstimulated and stimulated (BIMPS) hippocampal slices. Error bar represents s.e.m. $(n=4)$. Back-phosphorylation was reduced to $67 \pm 12 \%(P<0.05)$ of the control value, indicating that Snapin is phosphorylated by endogenous PKA in

Snapin protein immunoprecipitated by anti-Snapin antibody. Quantitative analysis of four experiments showed that BIMPS stimulation significantly reduced back-phosphorylation of Snapin to $67 \pm 12 \%$ of the control value $(n=4, P<0.05$; Fig. $6 \mathrm{~b})$. These results indicate that Snapin may be a direct target of cAMPdependent phosphorylation in vivo. We next invesigated the effect of PKA phosphorylation of Snapin in vivo on its association with synaptotagmin and the SNARE complex. We determined the extent of association of native Snapin with the SNARE complex and synaptotagmin by observing co-immunoprecipitation from hippocampal-slice lysates with an anti-SNAP-25 antibody. Activation by BIMPS of PKA in neurons significantly increased the interactions of both Snapin $(154 \pm 16 \%, n=7, P<0.02)$ and synaptotag$\min (151 \pm 9.8 \%, n=7, P<0.005)$ with the SNARE complex, relative to untreated hippocampal slices (Fig. 6c, d). This in vivo effect was also observed in cultured hippocampal cells (data not shown). Physiological relevance of Snapin phosphorylation by PKA. Although our biochemical results indicate that Snapin phosphorylation may modulate the interaction of synaptotagmin with the SNARE complex through a cAMP-dependent mechanism, it should be noted that these studies only show the biochemical end points of b

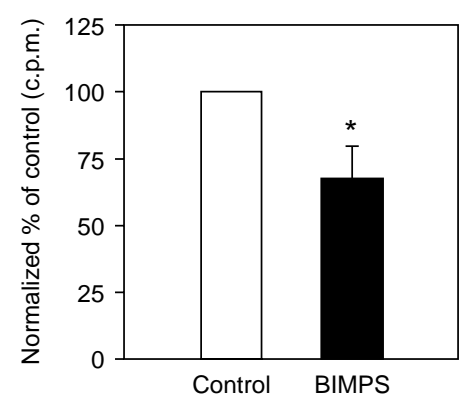

d
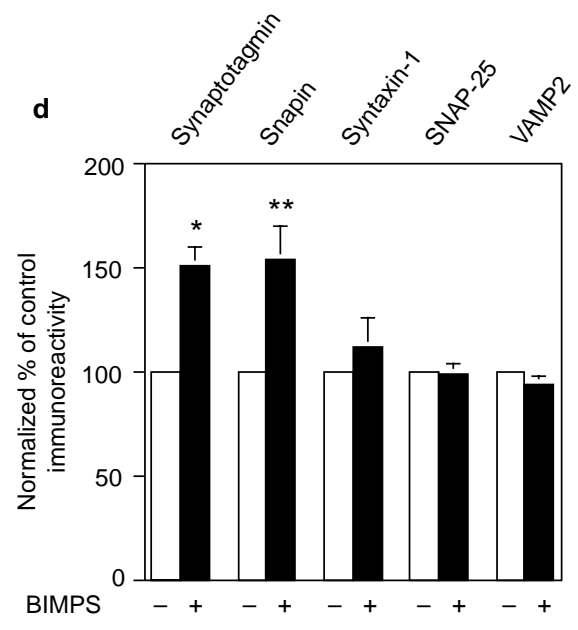

vivo in its native conformation. c, Stimulation of hippocampal neurons with cAMP analogue enhances the interactions of both Snapin and synaptotagmin with the SNARE complex. Total protein $(150 \mu \mathrm{g})$ extracted from stimulated (BIMPS) or unstimulated hippocampal slices was incubated with anti-SNAP25 antibody or normal IgG control. Co-precipitates were then analysed by sequential immunoblotting of the same membrane with antibodies against synaptotagmin, syntaxin-1, SNAP-25, VAMP2 and Snapin. Results are representative of seven experiments. d, Normalized amounts of protein immunoprecipitated by anti-SNAP-25 antibody from unstimulated (control) and stimulated (BIMPS) hippocampal-slice lysates. Values are means \pm s.e.m. from seven experiments. A two-tailed student's t-test for paired data was used; asterisk denotes $P<0.005$, double asterisk denotes $P<0.02$.

multiple protein interactions in vitro under solubilized conditions. Thus, the biochemical data alone do not allow us to examine protein interactions in the real-time scale of neurotransmitter release in vivo. To investigate the physiological effect of Snapin phosphorylation on transmitter release in vivo, we overexpressed the Snapin S50D mutant, which mimics a state of complete phosphorylation of native Snapin, and the Snapin S50A mutant, which mimics an unphosphorylated state of native Snapin, in bovine adrenal chromaffin cells. Chromaffin cells offer the unique opportunity to apply high-temporal-resolution techniques such as measurement of membrane capacitance and electrochemical detection of catecholamine release, thus allowing exocytosis to be studied in the millisecond range ${ }^{35}$. These measurements reveal the presence of different kinetic phases of secretion. A rapid increase in intracellular $\mathrm{Ca}^{2+}$ leads to an exocytotic burst followed by a sustained phase of secretion. Using flash photolysis of caged calcium as a fast stimulus, we studied the effect of the Snapin mutants on the different components of exocytosis. Overexpression of S50D Snapin resulted in a marked increase in the magnitudes of both the exocytotic burst (S50D Snapin, $468 \pm 60 \mathrm{fF}$; control, $347 \pm 33 \mathrm{fF}$ ) and the sustained component (S50D Snapin, $189 \pm 31 \mathrm{fF}, n=19$; control, $99 \pm 27 \mathrm{fF}, n$ 


\section{articles}
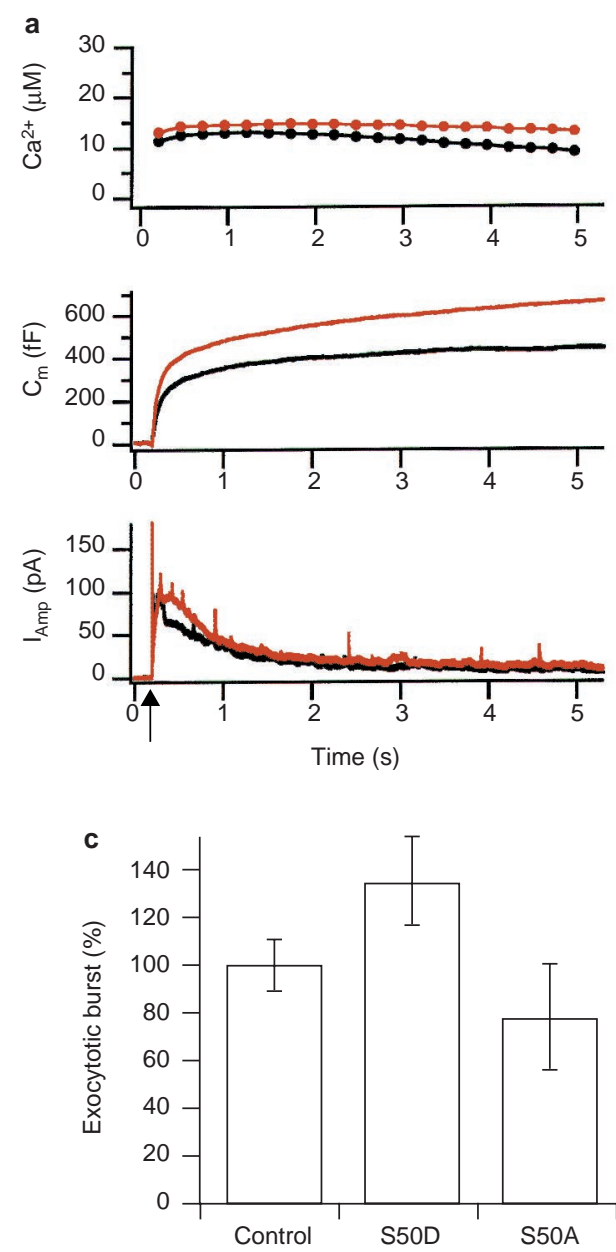

Figure 7 Physiological effects of overexpression of S50D and S50A Snapin on large dense-core vesicle (LDCV) exocytosis. a, b, Averaged high-temporalresolution recordings of membrane capacitance $\left(\mathrm{C}_{\mathrm{m}}\right.$; middle traces in $\mathbf{a}$ and $\left.\mathbf{b}\right)$ and amperometric current ( $\left(_{\text {Amp }}\right.$; lower traces in $\mathbf{a}$ and $\left.\mathbf{b}\right)$ in response to flash photolysis of caged calcium (indicated by arrow) from control cells (black; $n=18$ in $\mathbf{a}$ and $n=$ 14 in $\mathbf{b}$ ) and from cells overexpressing S50D Snapin (red in $\mathbf{a} ; n=19$ ) or S50A Snapin (red in $\mathbf{b} ; n=15$ ). For all cells, the intracellular calcium concentration was maintained at $\sim 15 \mu \mathrm{M}$ for $5 \mathrm{~s}$ (upper traces in $\mathbf{a}$ and $\mathbf{b}$ ). In comparison to control cells, cells overexpressing S50D Snapin exhibit increases in the magnitudes of both

$=18 ; P<0.05)$ relative to control cells $($ Fig. $7 \mathrm{a}, \mathrm{c})$. In contrast, overexpression of S50A Snapin led to a reduction in the magnitude of the burst component (S50A Snapin, $296 \pm 72 \mathrm{fF}$; control, $377 \pm 52$ $\mathrm{fF}$ ), whereas that of the sustained component was increased to a similar extent as in cells expressing S50D Snapin (S50A Snapin, 189 \pm 39 fF, $n=15$; control, $88 \pm 23 \mathrm{fF}, n=14 ; P<0.05$; Fig. $7 \mathrm{~b}, \mathrm{c})$. As both components of the exocytotic burst represent fusion-competent (primed) vesicles, these results demonstrate that phosphorylation of Snapin at serine 50 plays a positive role in the priming of large dense-core vesicle exocytosis.

\section{Discussion}

Our previous results provided biochemical and physiological evidence that Snapin is an important component of the neurotransmitter-release machinery, as it binds to SNAP-25 and consequently potentiates the interactions between the SNARE complex and synaptotagmin $^{29}$. Introduction of excess Snapin peptide fragments containing the SNAP-25-binding sequence into presynaptic superior
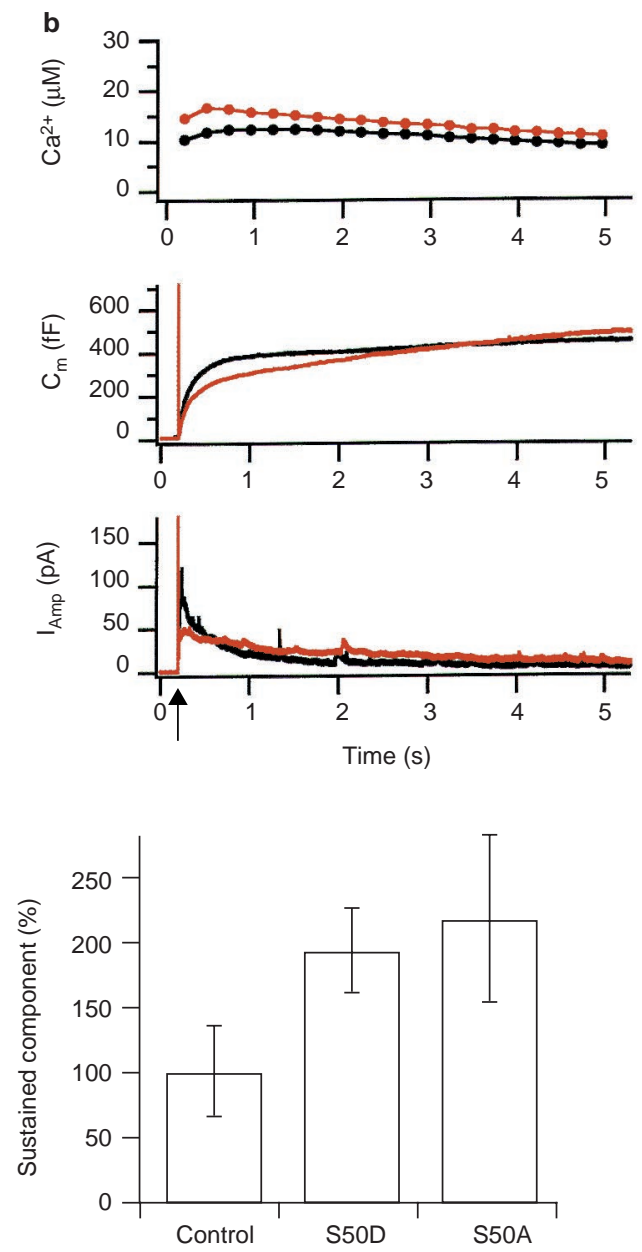

the exocytotic burst (up to $1 \mathrm{~s}$ after the flash) and the sustained component (1-5 s after the flash). Cells expressing S50A Snapin also exhibit a sustained component of increased magnitude, whereas that of the exocytotic burst is markedly reduced. c, Summary of the mean capacitance amplitude of the exocytotic burst (left) and of the sustained component of LDCV secretion for control cells and cells overexpressing S50A and S50D Snapin. To account for any putative variability in cell preparation, values for each overexpressed mutant are normalized to an independent batch of control cells.

cervical ganglion neurons (SCGNs) in culture effectively inhibited synaptic transmission. We interpreted this inhibitory effect as a competitive block of endogenous Snapin binding to SNAP-25 and a consequent reduction in association of the SNARE-synaptotagmin complex.

The results presented here indicate that Snapin can serve as a target for PKA at the synapse to modulate transmitter release and neuronal plasticity through a second-messenger pathway. Phosphorylation of Snapin at serine 50, both in vitro and in vivo, leads to an increase in binding of Snapin to SNAP-25. Furthermore, this phosphorylation apparently strengthens the association of synaptotagmin, a proposed $\mathrm{Ca}^{2+}$ sensor for exocytosis, with the assembled SNARE complex. In physiological experiments, overexpression of constitutively phosphorylated Snapin (S50D Snapin) leads to an increase in the number of release-ready vesicles in adrenal chromaffin cells, whereas overexpression of the unphosphorylated form (S50A Snapin) reduces this number. In the context of the current model of exocytosis in chromaffin cells ${ }^{36}$, our findings lead to the following conclusions: first, both the S50D and S50A 
mutations increase the magnitude of the sustained component of exocytosis, indicating that Snapin acts as a positive modulator of the priming step by increasing the forward rate constant of priming ${ }^{36}$. Second, S50D Snapin increases the size of the exocytotic burst, wheresa S50A Snapin reduces it. Thus, phosphorylation of Snapin leads to stabilization of release-ready vesicles, most probably by reducing the backward rate constant for the priming-unpriming reaction ${ }^{36}$.

Our current hypothesis regarding the molecular mechanism that underlies the priming step is that it represents the formation of a SNARE complex consisting of SNAP-25, syntaxin and synaptobrevin ${ }^{37}$. Our physiological data seem to indicate that Snapin facilitates formation of the SNARE complex, and that PKA phosphorylation of Snapin stabilizes the formed complexes. However, as primed vesicles in chromaffin cells are ready to fuse upon elevation of $\mathrm{Ca}^{2+}$ concentration, it remains possible that the priming step also involves association of a $\mathrm{Ca}^{2+}$ sensor, such as synaptotagmin, with the SNARE complex. Further experiments are needed to evaluate the exact molecular mechanism that underpins the stimulatory role of Snapin in exocytosis.

It has previously been shown that other synaptic proteins are substrates for PKA. PKA phosphorylation of $\alpha$-SNAP markedly reduces its ability to bind to the $7 \mathrm{~S}$ core complex, consequently resulting in a reduction in $\mathrm{N}$-ethylmaleimide-sensitive factor (NSF) function $^{32}$. A reduction in NSF function would result in lower levels of vesicle fusion and neurotransmitter release. Therefore, on the basis of the current SNARE hypothesis, $\alpha$-SNAP is probably not a primary target for PKA modulation in facilitating synaptic transmission. We and others have shown that SNAP-25 is an efficient substrate for PKA and that the site of PKA phosphorylation in this protein is threonine 138, which lies in the core of the SNARE complex and is therefore not accessible in native SNARE complexes ${ }^{31}$. This phosphorylation event does not influence the interactions of SNAP-25 with syntaxin-1A, VAMP-2, synaptotagmin or Snapin, and therefore does not directly regulate the assembly of functional SNARE complexes. Rabphilin-3A, a synaptic-vesicle protein that interacts with rab3A in a GTP-dependent manner, is an efficient substrate for PKA in vitro ${ }^{38}$. Forskolin increases the phosphorylation of rabphilin in CA3/mossy-fibre synaptosomes, but not in CA1 synaptosomes ${ }^{39}$, indicating that it may be a PKA target for inducing mossy-fibre long-term potentiation in the hippocampus. However, it has been shown that forskolin can facilitate synaptic transmission in the hippocampus of Rab3A-deficient mice ${ }^{40}$, which exhibit a significant reduction in rabphilin-3A levels in most synapses ${ }^{41,42}$. Thus, it is still unclear whether phosphorylation of rabphilin-3A by PKA has a direct influence on synaptic transmission. Recent studies have shown that forskolin has several effects on release of glutamate from hippocampal neurons by augmenting the size of the readily releasable pool of vesicles, increasing the ability of membrane depolarization to evoke release, and potentiating $\mathrm{Ca}^{2+}$ activity in exocytosis ${ }^{43}$. Our physiological results, in which we observed an increase in the number of release-competent vesicles upon overexpression of S50D Snapin, are consistent with the findings in these studies. Our data demonstrate for the first time that phosphorylation of a single protein - Snapin - can influence a particular step - priming - in the presynaptic vesicle cycle. However, it remains possible that PKA acts on other synaptic regulatory proteins, either directly or indirectly, by activating downstream protein kinases and thereby further modifies the physiology of synaptic transmission.

We have highlighted the potential importance of phosphorylation of Snapin by PKA for regulation of synaptic vesicle exocytosis. We have provided the foundation for further studies aimed at understanding the structural and functional aspects of these events. Using phosphopeptide-specific antisera raised against the serine 50 phosphorylation site and examining spatial and temporal signal activation of this regulatory pathway in vivo will allow us to gain a better understanding of the molecular mechanisms that underlie PKA-dependent synaptic plasticity.

\section{Methods}

\section{Preparation of fusion proteins.}

Full-length and truncated mutants of Snapin were subcloned into the hexahistidine-tagged fusionprotein vector (pET28C or A, Novagen, Madison, Wisconsin). Wild-type and substitution mutants of full-length Snapin, SNAP-25 and SNAP-23 were subcloned into the pGEX-4T vector (Pharmacia, Piscataway, New Jersey). Fusion proteins were prepared and further purified as described ${ }^{44}$. His-Snapin proteins were purified by binding to $\mathrm{Ni}^{2+}$-charged nitrilotriacetic acid-agarose columns (Qiagen), and were eluted with $500 \mathrm{mM}$ imidazole in PBS. GST-fusion proteins were purified by binding to glutathione-sepharose beads (Pharmacia) in TBS buffer (50 mM Tris-HCl pH 7.5, $140 \mathrm{mM} \mathrm{NaCl}, 0.1 \%$ Triton X-100 and protease inhibitors) and were cleaved from the GST tag by incubation with biotinylated thrombin (Novagen) at a final concentration of 0.1 units per $\mu \mathrm{l}$ at room temperature for $6 \mathrm{~h}$, or were eluted from beads in elution buffer ( $15 \mathrm{mM}$ reduced glutathione, $50 \mathrm{mM}$ Tris- $\mathrm{HCl}, \mathrm{pH} 8)$. Biotinylated thrombin was removed from the resulting cleavage reactions using streptiavidin-agarose beads (Novagen).

\section{Phosphorylation reactions.}

The 35- $\mu 1$ reaction mixture for in vitro PKA phosphorylation contained 50-100 pmol of purified Snapin or SNAP-25, PKA-activation buffer $(40 \mathrm{mM}$ Tris- $\mathrm{HCl} \mathrm{pH} 7.4$ and $20 \mathrm{mM}$ magnesium oxyacetate) and $80 \mu \mathrm{M}$ ATP in the presence or absence of $100 \mu \mathrm{g}$ pseudosubstrate peptide inhibitor PKI (Promega). To detect ${ }^{32} \mathrm{P}$ incorporation, $0.125 \mu \mathrm{M}\left[\gamma^{32} \mathrm{P}\right] \mathrm{ATP}\left(3,000 \mathrm{Ci} \mathrm{mmol}^{-1}\right.$; Amersham) was added. Samples to be phosphorylated were pre-incubated for $10 \mathrm{~min}$ at $30^{\circ} \mathrm{C}$ before addition of the catalytic subunit of PKA (Promega) to a final concentration of 0.6 units per $\mu$ l. Reactions were incubated for $30 \mathrm{~min}$ at $30^{\circ} \mathrm{C}$. For gel analysis, reactions were terminated by adding $30 \mu \mathrm{l}$ SDS-PAGE sample buffer; samples were then heated at $95^{\circ} \mathrm{C}$ for $3-5 \mathrm{~min}$. Aliquots of phosphorylated products were analysed by $10-20 \%$ Tricine SDS-PAGE. Gels were stained with Coomassie blue and were then dried and exposed to X-ray film for autoradiography.

\section{Stoichiometric measurements.}

For phosphorylation time-course experiments, 15 pmol of purified recombinant Snapin was cleaved from the GST tag in phosphorylation buffer (see above), supplemented with $0.352 \mu \mathrm{M}\left[\gamma-{ }^{32} \mathrm{P}\right] \mathrm{ATP}$, $0.352 \mathrm{mM}$ cold ATP and $1.5 \mu \mathrm{g}$ PKA, and incubated at $32{ }^{\circ} \mathrm{C}$ for between 5 and $240 \mathrm{~min}$. Reaction were terminated by addition of SDS sample buffer and boiling. After SDS-PAGE, staining with Coomassie blue, gel drying and autoradiography, relevant gel slices were excised and scintillationcounted to determine total ${ }^{32} \mathrm{P}$ incorporation. Specific radioactivity per mol $\left[\gamma-{ }^{32} \mathrm{P}\right] \mathrm{ATP}$ was determined by counting diluted aliquots of the stock, and this conversion value was used to calculate the molar amount of ${ }^{32} \mathrm{P}$ incorporated and the molar ratio of ${ }^{32} \mathrm{P}$ to Snapin protein.

\section{Site-directed mutagenesis.}

Snapin mutants were generated in a standard two-step megaprimer polymerase chain reaction (PCR) using Taq polymerase with mutated oligonucleotides as primers and full-length Snapin complementary DNA as a template. The first step included two PCR reactions that span the mutation site. Final PCR products were cloned into the pGEX-4T vector (Pharmacia), and mutations were verified by DNA sequencing.

\section{In vitro binding studies.}

GST-fusion proteins $(1 \mu \mathrm{g})$ immobilized on glutathione-sepharose beads were incubated with Snapin or phosphorylated Snapin in TBS buffer with $0.1 \%$ Triton X-100 for $5 \mathrm{~h}$ at $4{ }^{\circ} \mathrm{C}$. Beads were washed three times with incubation buffer, and bound proteins were eluted in $30 \mu \mathrm{l}$ SDS-PAGE sample buffer and processed for 10-20\% Tricine SDS-polyacrylamide gradient gel electrophoresis. Separated proteins were sequentially detected using a polyclonal anti-Snapin antibody and a monoclonal anti-GST antibody (Pharmacia) on the same blot. Horseradish-peroxidase-conjugated secondary antibodies and enhanced chemiluminescence (ECL, Amersham) were used to visualize bands. For quantitative analysis, binding intensities were scanned into NIH Image 1.61. Care was taken during exposure of the ECL film to ensure that all readings were in the linear range of a standard. The percentage of binding relative to that of unphosphorylated or wild-type Snapin was calculated using standard curves. Student $t$-tests were carried out and results are expressed as means \pm s.e.m. from three or six independent experiments.

\section{Preparation of synaptosomes and immunoprecipitation.}

Rat-brain synaptosomes were prepared by differential and discontinuous percoll-gradient centrifugation and were solubilized as described ${ }^{45,46}$. Briefly, rat-brain cortices or whole brains were homogenized in cold $21.9 \%$ sucrose buffer ( $\mathrm{pH} 7.4$ ) with protease inhibitors. Homogenates were centrifuged in a Beckman GSR-6RHT centrifuge at 3,000 r.p.m. for $10 \mathrm{~min}$. The supernatant was placed on top of percoll gradients $(23 \%, 15 \%, 10 \%$ and $3 \%$ in sucrose buffer), and spun in an SS34 rotor at 17,250 r.p.m. for $5 \mathrm{~min}$. The synaptosome band between the $15 \%$ and $23 \%$ gradients was collected, mixed with TBS, and then spun in an SS34 rotor at 11,000 r.p.m. for $15 \mathrm{~min}$. The resulting synaptosome pellet was resuspended in solubilization buffer $(150 \mathrm{mM} \mathrm{NaCl}, 10 \mathrm{mM}$ HEPES pH 7.2, $1.2 \%$ Triton X-100 and protease inhibitors) and stirred at $4{ }^{\circ} \mathrm{C}$ for $1 \mathrm{~h}$. The solution was centrifuged at 13,000 r.p.m. in a Beckman F2402H rotor for $1 \mathrm{~h}$. The supernatant that was enriched for solubilized synaptic proteins was collected. Solubilized proteins $(100-300 \mu \mathrm{g})$ from synaptosome preparations were incubated with either $1 \mu \mathrm{g}$ anti-SNAP-25 (BR05) monoclonal antibody or mouse control immunoglobulin $\mathrm{G}$ in $250 \mu \mathrm{l}$ TBS with $0.1 \%$ Triton X-100, $0.1 \% \mathrm{BSA}, 200 \mu \mathrm{M} \mathrm{CaCl}_{2}$ and protease inhibitors at $4{ }^{\circ} \mathrm{C}$ for $1 \mathrm{~h}$, in the presence or absence of the indicated amounts of wild-type or mutant Snapin. Protein A-sepharose CL$4 \mathrm{~B}$ resin ( $2.5 \mathrm{mg}$; Pharmacia) was added to each sample, and incubation was continued for a further 3 $\mathrm{h}$, after which samples were washed three times with TBS/0.1\% Triton X-100. Subsequent interaction assays of immunoprecipitated and immobilized protein complexes are described under ' $I n$ vitro binding studies'. For multiple detection with different antibodies, blots were first stripped in a solution of $62.5 \mathrm{mM}$ Tris- $\mathrm{HCl} \mathrm{pH} \mathrm{7.5,} 20 \mathrm{mM}$ dithiothreitol and $1 \%$ SDS for $20-30 \mathrm{~min}$ at $50^{\circ} \mathrm{C}$, and were then washed with TBS $/ 0.1 \%$ Tween- 20 for $2 \times 15 \mathrm{~min}$. 


\section{Preparation and treatment of hippocampal slices.}

Hippocampal slices $(400 \mu \mathrm{m})$ were prepared from 4-week-old male Sprague-Rawley rats as described ${ }^{47,48}$. Slices were prepared in ice-cold artificial cerebrospinal fluid (ACSF) containing (in $\mathrm{mM}$ ) $119 \mathrm{NaCl}, 2.5 \mathrm{KCl}, 1.3 \mathrm{MgCl}_{2}, 26.2 \mathrm{NaHCO}_{3}, 11$ glucose and $2.5 \mathrm{CaCl}_{2}$, bubbled with $95 \% \mathrm{O}_{2}$ and $5 \%$ $\mathrm{CO}_{2}$. Slices were then transferred to identical solution at $37^{\circ} \mathrm{C}$ for $30 \mathrm{~min}$ and were then maintained at room temperature for a further $30 \mathrm{~min}$. Paired slices were then incubated at $37^{\circ} \mathrm{C}$ in the presence or absence of $50 \mu \mathrm{M}$ of the cAMP analogue Sp-5,6-DCl-cBIMPS (BIMPS), and were then lysed in lysis buffer (1.2\% Triton X-100, $5 \mathrm{mM}$ EDTA, $10 \mathrm{mM}$ Tris, $50 \mathrm{mM} \mathrm{NaCl}$, the following phosphatase inhibitors (in $\mathrm{mM}$ ): $20 \beta$-glycerol phosphate, $50 \mathrm{NaF}, 50$ sodium pyrophosphate and 1 p-nitrophenyl phosphate, and $0.2 \mu \mathrm{M}$ microcystin-LR) on ice for $30 \mathrm{~min}$. Cell lysates were centrifuged $(15,000 \mathrm{~g}, 20$ $\mathrm{min})$ and then ultracentrifuged $(40,000 \mathrm{~g}, 1 \mathrm{~h})$ to remove insoluble material.

\section{Electrophysiological measurements.}

Chromaffin cells were prepared and infected with pSFV1-Snapin S50A and S50D IRES mutants as described ${ }^{49}$. Experiments were carried out $18-36 \mathrm{~h}$ after infection at $30-32{ }^{\circ} \mathrm{C}$. The external bathing solution contained (in mM): $140 \mathrm{NaCl}, 2.8 \mathrm{KCl}, 2 \mathrm{CaCl}_{2}, 1 \mathrm{MgCl}_{2}$ and $10 \mathrm{HEPES}$, and $2 \mathrm{mg} \mathrm{ml}^{-1} \mathrm{glu}-$ cose ( $\mathrm{pH} 7.2,310-320 \mathrm{mOsm}$ ). The internal pipette solution contained (in mM): 105 caesium glutamate, 2 MgATP, 0.3 GTP, 33 HEPES and 0.33 Fura2-FF, adjusted to $\mathrm{pH} 7.2$ with $\mathrm{HCl}$ or caesium hydroxide (300-310 mOsm). The basal $\left[\mathrm{Ca}^{2+}\right]_{\mathrm{i}}$ was buffered using a combination of $4 \mathrm{mM} \mathrm{CaCl}_{2}$ and 5 $\mathrm{mM}$ nitrophenyl-EGTA to give a free $\left[\mathrm{Ca}^{2+}\right]_{\mathrm{i}}$ of $200-300 \mathrm{nM}$ (ref. 36). Amperometric measurements and flash photolysis of caged calcium were carried out as described ${ }^{49}$; data are espressed as means \pm s.e.m.

RECEIVED 20 DECEMBER 1999; REVISED 7 NOVEMBER 2000; ACCEPTED 13 DECEMBER 2000; PUBLISHED 6 MARCH 2001.

1. Rothman, J. E. Mechanisms of intracellular protein transport. Nature 372, 55-63 (1994).

2. Bajjalieh, S. M. \& Scheller, R. H. The biochemistry of neurotransmitter secretion. J. Biol. Chem. 270, 1971-1974 (1995).

3. Südhof, T. C. The synaptic vesicle cycle: a cascade of protein-protein interactions. Nature 375 , 645-653 (1995).

4. Hilfiker, S., Greengard, P. \& Augustine, G. J. Coupling calcium to SNARE-mediated synaptic vesicle fusion. Nature Neuroscience 2, 104-106 (1999).

5. Trimble, W. S., Cowan, D. M. \& Scheller, R. H. VAMP-1: a synaptic vesicle-associated integral membrane protein. Proc. Natl Acad. Sci. USA 85, 4538-4542 (1988).

6. Bennett, M. K., Calakos, N. \& Scheller, R. H. Syntaxin: a synaptic protein implicated in docking of synaptic vesicles at presynaptic active zones. Science 257, 255-259 (1992).

7. Oyler, G. A. et al. The identification of a novel synaptosomal-associated protein, SNAP-25, differentially expressed by neuronal subpopulations. J. Cell. Biol. 109, 3039-3052 (1989).

8. Söllner, T. et al. SNAP receptors implicated in vesicle targeting and fusion. Nature 362, 318-324 (1993).

9. Calakos, N., Bennett, M. K., Peterson, K. \& Scheller, R. H. Protein-protein interactions contributing to the specificity of intracellular vesicular trafficking. Science 263, 1146-1149 (1994).

10. Hayashi, T. et al. Synaptic vesicle membrane fusion complex: action of clostridial neurotoxins on assembly. EMBO J. 13, 5051-5061 (1994).

11. Weber, T. et al. SNAREpins: minimal machinery for membrane fusion. Cell 92, 759-772 (1998).

12. Chen, Y. A., Scales, A. J., Patel, S. M., Doung, Y.-C. \& Scheller, R. H. SNARE complex formation is triggered by $\mathrm{Ca}^{2+}$ and drives membrane fusion. Cell 97, 165-174 (1999).

13. Bommert, K. et al. Inhibition of neurotransmitter release by C2-domain peptides implicates synaptotagmin in exocytosis. Nature 363, 163-165 (1993).

14. Broadie, K., Bellen, H. J., DiAntonio, A., Littleton, J. T. \& Schwarz, T. L. Absence of synaptotagmin disrupts excitation-secretion coupling during synaptic transmission. Proc. Natl Acad. Sci. USA 91, 10727-10731 (1994).

15. Geppert, M. et al. Synaptotagmin I: a major $\mathrm{Ca}^{2+}$ sensor for transmitter release at a central synapse. Cell 79, 717-727 (1994).

16. Bliss, T. V. P. \& Collingridge, G. L. A synaptic model of memory: long-term potentiation in the hippocampus. Nature 361, 31-39 (1993).

17. Nicoll, R. A. \& Malenka, R. C. Contrasting properties of two forms of long-term potentiation in the hippocampus. Nature 377, 115-118 (1995).

18. Martinez, J. L. Jr \& Derrick, B. E. Long-term potentiation and learning. Annu. Rev. Psychol. 47, $173-203$ (1996).

19. Bouron, A. \& Reuter, H. Muscarinic stimulation of synaptic activity by protein kinase $\mathrm{C}$ is inhibited by adenosine in cultured hippocampal neurons. Proc. Natl Acad. Sci. USA 94, 12224-12229 (1997).

20. Dixon, D. \& Atwood, H. L. Adenylated cyclase system is essential for long-term facilitation at the crayfish neuromuscular junction. J. Neurosci. 9, 4245-4252 (1989).

21. Chavez-Noriega, L. E. \& Stevens, C. F. Increased transmitter release at excitatory synapses produced by direct activation of adenylated cyclase in rat hippocampal slices. J. Neurosci. 14, 310-317 (1994)

22. Klein, M. Synaptic augmentation by 5-HT at rested Aplysia sensorimoter synapses: independence of action potential prolongation. Neuron 13, 159-166 (1994).

23. Capogna, M., Gähwiler, B. H. \& Thompson, S. M. Presynaptic enhancement of inhibitory synaptic transmission by protein kinases A and C in the rat hippocampus in vitro. J. Neurosci. 15, 1249-1260 (1995).

24. Chavis, P., Mollard, P., Bockaert, J. \& Manzoni, O. Visualization of cyclic AMP-regulated presynaptic activity at cerebellar granule cells. Neuron 20, 773-781 (1998).

25. Huang, Y. Y., Li, X. C. \& Kandel, E. R. cAMP contributes to mossy fiber LTP by inititating both a covalently mediated early phase and a macromolecular synthesis-dependent late phase. Cell 79, 69-79 (1994).

26. Weisskopf, M. G., Castillo, P. E., Zalutsky, R. A. \& Nicoll, R. A. Mediation of hippocampal mossy fiber long-term potentiation by cyclic-AMP. Science 265, 1876-1882 (1994).

27. Trudeau, L. E., Emery, D. G. \& Haydon, P. G. Direct modulation of the secretory machinery underlies PKA-dependent synaptic facilitation in hippocampal neurons. Neuron 17, 789-797 (1996).

28. Trudeau, L. E., Fang, Y., \& Haydon, P. G. Modulation of an early step in the secretory machinery in hippocampal nerve terminals. Proc. Natl Acad. Sci. USA 95, 7163-7168 (1998).

29. Ilardi, J. M., Mochida, S., \& Sheng, Z.-H. Snapin: a SNARE-associated protein implicated in synaptic transmission. Nature Neurosci. 2, 119-124 (1999).

30. Kennelly, P. J. \& Krebs, E. G. Consensus sequences as substrate specificity determinants for protein kinases and protein phosphatases. J. Biol. Chem. 266, 15555-15558 (1991).

31. Risinger, C. \& Bennett, M. K. Differential phosphorylation of syntaxin and synaptosome-associated protein of $25 \mathrm{kDa}$ (SNAP-25) isoforms. J. Neurochem. 72, 614-624 (1999).

32. Hirling, H. \& Scheller, R. H. Phosphorylation of synaptic vesicle proteins: modulation of the aSNAP interaction with the core complex. Proc. Natl Acad. Sci. USA 93, 11945-11949 (1996).

33. Foster, L. J. et al. Binary interactions of the SNARE proteins syntaxin-4, SNAP23, and VAMP-2 and their regulation by phosphorylation. Biochem. 37, 11089-11096 (1998).

34. Sandberg, M. et al. Characterization of Sp-5,6-dichloro-1- $\beta$-D ribofuranosylbenzimidazole-3', 5'monophosphorothioate (Sp-5,6-DCl-cBIMPS) as a potent and specific activator of cyclic AMPdependent protein kinase in cell extracts and intact cells. Biochem. J. 279, 521-527 (1991).

35. Neher, E. Vesicle pools and $\mathrm{Ca}^{2+}$ microdomains: new tools for understanding their roles in neurotransmitter release. Neuron 20, 389-399 (1998).

36. Ashery, U. et al. Munc13-1 acts as a priming factor for large-dense core vesicles in bovine chromaffin cells. EMBO J. 19, 3586-3596 (2000).

37. Brose, N., Rosenmund, C. \& Rettig, J. Regulation of transmitter release by Unc-13 and its homologues. Curr. Opin. Neurobiol. 10, 303-311 (2000).

38. Fykse, E. M., Li, C. \& Südhof, T. C. Phosphorylation of rabphilin-3A by $\mathrm{Ca}^{2+} / \mathrm{calmodulin}^{-}$and cAMP-dependent protein kinases in vitro. J. Neurosci. 15, 2385-2395 (1995).

39. Lonart, G. \& Südhof, T. C. Region-specific phosphorylation of rabphilin in mossy fiber nerve terminals of the hippocampus. J. Neurosci. 18, 634-640 (1998).

40. Castillo, P. E. et al. The synaptic vesicle protein rab3A is essential for mossy fiber long term potentiation in the hippocampus. Nature 388, 590-593 (1997).

41. Geppert, M. et al. Rab3A functions in neurotransmitter release. Nature 369, 493-497 (1994).

42. Li, C. et al. Synaptic targeting of rabphilin-3A, a synaptic vesicle $\mathrm{Ca}^{2+} /$ phospholipid-binding protein, depends on rab3A/3C. Neuron 13, 885-898 (1994).

43. Lonart, G., Janz, R., Johnson, K. M. \& Südhof, T. C. Mechanism of action of rab3A in mossy fiber LTP. Neuron 21, 1141-1150 (1998)

44. Yokoyama, C. T., Sheng, Z-H., \& Catterall, W. A. Phosphorylation of the synaptic protein interaction site on $\mathrm{N}$-type calcium channels inhibits interactions with SNARE proteins. J. Neurosci. 17, 6929-6938 (1997).

45. Sheng, Z-H., Rettig, J., Cook, T. \& Catterall, W. A. Calcium-dependent interaction of N-type calcium channels with the synaptic core complex. Nature 379, 451-454 (1996).

46. Dunkley, P. R. et al. A rapid percoll gradient procedure for isolation of synaptosomes directly from an S1 fraction: homogeneity and morphology of subcellular fractions. Brain Res. 441, 59-71 (1988).

47. Yan, Z., Feng, J., Fienberg, A. A. \& Greengard, P. D2 dopamine receptors induce mitogen-activated protein kinase and cAMP response element-binding protein phosphorylation in neurons. Proc. Natl Acad. Sci. USA 96, 11607-11612 (1999).

48. Diamond, J. S., Bergles, D. E. \& Jahr, C. E. Glutamate release monitored with astrocyte transporter currents during LTP. Neuron 21, 425-433 (1998).

49. Ashery, U., Betz, A., Xu, T., Brose, N. \& Rettig, J. An efficient method for infection of adrenal chromaffin cells using the Semliki Forest virus gene expression system. Eur. J. Cell Biol. 78, 525-532 (1999).

ACKNOWLEDGMENTS

We thank Q. Su for constructs of pSFV1-Snapin mutants, C. M. Gerwin, A. Bührmann and F. Friedlein for technical assistance, X. Zheng for synaptsome preparations, J. S. Diamond for hippocampal slices, M. Takahashi for antibodies, and J. W. Nagle for DNA sequencing. We also thank H. Gainer, C. T.

Yokoyama, S. Das and J. Coulombe for comments on the manuscript, and E. Neher for continuous support. This work was supported by the intramural research program of NINDS, NIH (to Z-H. S.), the Deutsche Forschungsgemeinschaft (to J.R.), an EU Network Grant (to U.A.), and the HHMI-NIH Research Scholars Program (to M.G.C.).

Correspondence and requests for materials should be addressed to Z-H.S. 